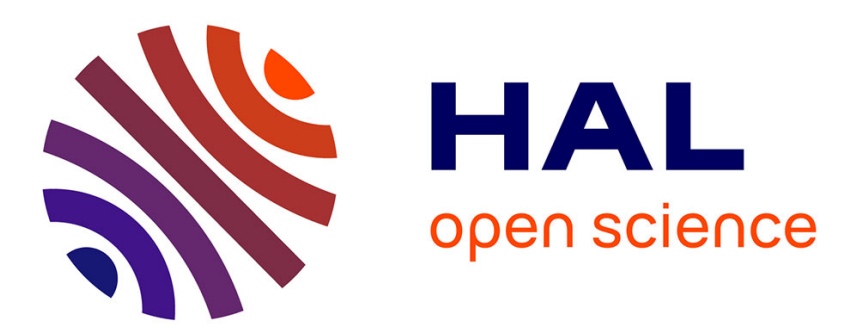

\title{
Localisation of Thermoelastoplastic Deformations in the Case of Simple Shear.
}

\author{
P. Guélin, W. Nowacki, H. Nguyen
}

\section{To cite this version:}

P. Guélin, W. Nowacki, H. Nguyen. Localisation of Thermoelastoplastic Deformations in the Case of Simple Shear.. Journal de Physique IV Proceedings, 1997, 07 (C3), pp.C3-717-C3-722. 10.1051/jp4:19973122 . jpa-00255408

\section{HAL Id: jpa-00255408 https://hal.science/jpa-00255408}

Submitted on 1 Jan 1997

HAL is a multi-disciplinary open access archive for the deposit and dissemination of scientific research documents, whether they are published or not. The documents may come from teaching and research institutions in France or abroad, or from public or private research centers.
L'archive ouverte pluridisciplinaire HAL, est destinée au dépôt et à la diffusion de documents scientifiques de niveau recherche, publiés ou non, émanant des établissements d'enseignement et de recherche français ou étrangers, des laboratoires publics ou privés. 


\title{
Localisation of Thermoelastoplastic Deformations in the Case of Simple Shear.
}

\author{
P. Guélin*, W.K. Nowacki and H.V. Nguyen
}

Center of Mechanics, Institute of Fundamental Technological Research of Polish Academy of Sciences, ul. Swietokrzyska 21, 00-049 Warsaw, Poland

*Laboratoire 3S, U.M.R. C.N.R.S., Université J. Fourier, BP. 53, 38041 Grenoble cedex 9, France

\begin{abstract}
The results of quasistatic and dynamic plane shear test are discussed. The temperature changes occurring during the shear have been recorded. The constitutive relations are formulated for elastic-plastic metallic solids at finite strain, for an adiabatic process with combined isotropic-kinematic hardening. The initial-boundaryvalue problem associated with simple shear thermomechanical experiment is formulated and solved. If the strain remains below $70 \%$ a remarkable homogeneity of the remnant strain and of the temperanure field is obtained. through the experiment as well as trough the numerical results. Beyond this strain value the localisation is observed.

Résumé. Les résultats đ'essais quasi-statiques et dynamiques de cisaillement simple sont discutés. Les changement de température associés ont été enregistrés. Des relations constitutives pour solides métalliques avec écrouissage isotrope et cinématique sont présentées en incluant le traitement des déformations finies. On suppose que le processus est adiabatique. Le problème aux valeurs initiales et aux limites associè à cette expérience thermomécanique est posé et résolu. Si la déformation reste inférieure à $70 \%$ une homogénéité exceptionnelle des champs de déformation rémanente et de température est obtenuc. à la fois dans l'expérience et comme résultat de la simulation numérique. Au-delà de $70 \%$, la localisation est observée.
\end{abstract}

\section{INTRODUCTION}

It is often useful to implement a variety of numerical systems in order to simulate the mechanical behaviour of thin-walled constructions, such as body of automobiles, buses, shells of wagons, air-planes, etc., subjected to the impact loadings. It is then necessary to know the dynamical behaviour of the thin sheets which are the main component involved in the structures. Owing to the fact that their mechanical characteristics are dependent of the metallurgical composition of the metal as well of the manner on its production, it is necessary to obtain with the aid of relevant experiments the data concerning this specific form of materials. Simple shear tests are of significant importance for the experimental investigation of the constitutive equations of materials. This type of test may be considered as complementary to the usual tests based on kinematics of tension or compression type or of pure shear type. However, the plane shear testing method presents some intrinsic drawbacks proceeding from the limit conditions. On one hand the clamping of the specimen is certainly not perfect and some sliding occurs between the specimen and the grips. On the other hand the shear stresses and strains vanish at the two free ends of specimen, introducing then an heterogeneity in the stress and strain states.

A set of quasistatic pure shear cyclic test has been performed recently in order to study the modelling of a material with the aid of an elastic-plastic pattern involving the discrete memory notion [1], [2]. In this case the pure shear of a plane specimen is obtained with the help of a special shear device. This shear device is essentially composed of a very rigid frame constituted by a rectangular box with a partial cover, inside which moves a vertical carriage. One specimen clamp is bound to the frame, as the other clamp is bound to a horizontal carriage. Thus the decice is able to impose a plane pure shear test with no lateral force: the test is then similar to that of pure torsion type, performed on tubes without axial force [3].

More recently, a new shear device was used to perform tests under high strain rates on specimens having the form of slabs such as metal sheets [4]. The features of this device are recalled in the first part of the paper. The loading and the displacements of this device are controlled by a Split Hopkinson Pressure 
Bar (SHPB) acting in compression. The role of the special device is to transform the compression into a plane shear. The dynamic simple plane shear tests so obtained is the only known method allowing to obtain a very good homogeneity of the remnant strain field over the total length of the specimen, without the localisation of deformations as in the case of torsion of thin-walled tubes [3].

In the second part of this paper, the relevant initial-boundary-value problem of the simple shear is formulated, taking into account the case of finite deformations. The rate-independent constitutive relations are implemented in the case of adiabatic processes and with combined kinematic-isotropic hardening at moderate pressures. The analytical solution is compared with the experimental data. The performed numerical calculations enabled the evaluation of the optimal dimensions of the specimen used in the case of dynamic loading. It will be useful to study further the quantitative modelling of the thermal effects

\section{EXPERIMENT}

The shear device consists of two coaxial cylindrical parts, namely the external tubular part and the internal massive part. Both cylinders are divided into two symmetrical parts, and between them the sheet in testing is fixed. Two bands of the specimen between the internal and external parts of the device are in plane shear when these cylinders move axially one toward the other. Each band before test is rectangular and becomes very near parallelogram having the constant length and the constant height. The greatest specimen height allowed by the shear device is $l_{0}=30 \mathrm{~mm}$. The width value $a_{0}$ is chosen with a view to satisfying two requirements: first the ratio $a_{d} / d$ of the width to the thickness has to be small enough to avoid the buchling of the sheared zone and secondly the ratio $a_{\alpha} / l_{o}$ of the width to the height has to be sufficiently small to minimize the error due to the non homogeneity of the shear stress and strain at the two ends of the sample. In our case, the material sheet has a nominal thickness of 0.5 or $0.75 \mathrm{~mm}$. We take in our tests the ratio $a_{\delta} l_{o}=1 / 10$ and we suppose that the results of test are good for both static and dynamic cases [1].[4].

First, the system is tested under quasi-static loading in order to verify its effectiveness. The dynamic test is similar but the loading is realized by the SHPB. The device with specimen is placed between two bars of the SHPB. In this case the mechanical impedance of the shear device and the SHPB must be the same to avoid the noise in the interface signal. The impulse is created by the third projectile bar: the usual compression technique. We have to register the input, transmitted and reflected impulse: $\varepsilon_{i}, \varepsilon_{t}$ and $\varepsilon_{r}$.

The highest strain rate in the specimen can be obtained using only one bar in the SHPB system. We use the transmitted bar only and the shearing device is placed in the front of this bar. The projectile bar strikes directly in the device. We have to register only the transmitted impulse $\varepsilon_{t}$ and the velocity of the projectile.

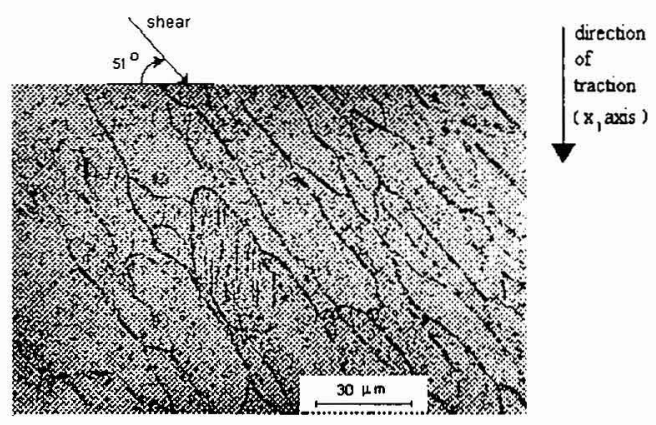

Fig. 1. Macro- and micro-bands of shear in the specimen

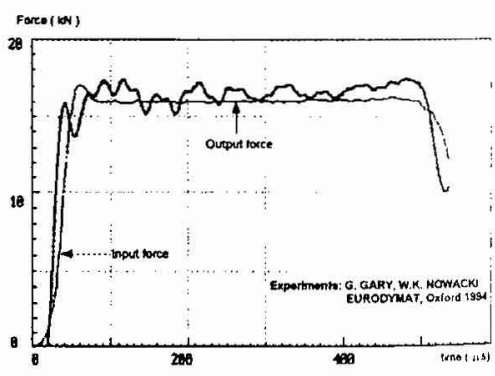

Fig.2, Measured input and output forces

The specimens deformed quasi-statically or dynamically to $70-90 \%$ as observed under the optical microscope, have a similar structure. The traversal lines marked on the specimens, on the gauge section. before the test remain parallel after the test. This fact indicate that the deformation is homogeneous in the considerable part of the specimen. However, the presence of free bounds of specimen produces the 
heterogeneity of stress field because the stress vector normal to the free surfaces must be zero, therefore we have assumed that the dimensions of the perturbed zone are small as compared to the dimensions of the specimen. The exceptional qualities of the homogeneity of the residual strain field show that the simplified analysis can be used in the zone of plastic deformations.

A portion of the gauge length of the specimen submitted to the simple shear test, with high strain rate, is shown in Fig. 1. The shear deformation is of the order of $73 \%$. An essential feature is the formation of the tangled structure and of the dislocations cells. Their elongation and arrangement tend to be aligned along the shear direction. In several grains the micro-bands of shear parallel to the direction of $x_{1}$ axis are observed. At the very high strain rate the shear macro-bands are observed. We can suppose that it is a critical strain at which the shear localization occurs. Before arriving at the critical strain, the deformation is homogeneous over the whole gauge length of the specimen. The work-hardening results from the creation, multiplication and interaction of the dislocations. In this case a small part of the work of plastic deformation is stored in the material as elastic strain energy (about 6\%) and the remaining part is converted into heat.

In the paper [5] the temperature field due to quasistatic plastic shear is measured (for the same steel sheets). The goal of this paper was to obtain the mechanical curves as well as the temperature distributions in the simple plane shear regions. A change of temperature of the surface of these regions has been observed by the thermovision camera coupled with a system of data acquisition and conversion. The infrared radiation emitted by shear paths was measured. The disturbances of the stress and strain fields existing on free ends of the specimen during shear test are visible in the temperature distribution. The temperature of these areas is higher than in remaining part of the specimen. This is easily seen in the initial stage of the process. At higher deformations this effect is difficult to observe because of large temperature increments of the shear zones and due to high heat conductivity of this steel. The results obtained enable us to present the temperature changes of the specimens subjected to the shear test with different rates of deformation, as well as to describe the macroscopic shear band which develops at higher deformations.

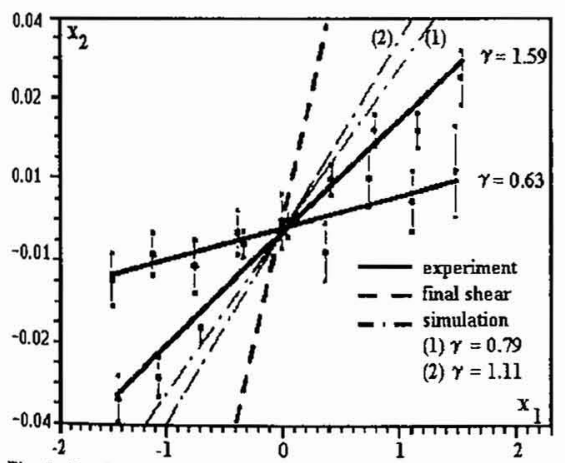

Fig. 3. Position of maximum temperature in shear zones

This macroscopic shear band develops at a certain angle to the shear path. Results (with linear approximation) were marked in the Fig. 3 by solid lines, while the dashed line show the position of the macroscopic shear band corresponding to final form of shear. Our results would indicate the change of the slope of macroscopic shear band with the deformation. At small temperature increments it can cause an effect of apparent change of inclination of the macroscopic shear band. Moreover, larger slope of this band observed at higher rate of shear, would indicate this type of effect. Suitable processing of thermal pictures recorded during

the shear test indicates, that the phenomenon of localized deformation is noticeable also in nonadiabatic conditions, in which the experiment has been conducted. With this technique, it is possible to evaluate the stored energy due to the simple shear in the case of large deformations.

In the analysis, we must take into account that the loading of the specimen is not instantaneous. The loading compression wave must take some time to transmit from one end of the device to the other. However, it is worthwhile to notice that, in our tests, a very accurate equilibrium is obtained regarding the forces on two sides of the shear device, see Fig. 2 . We observe that the input force and the output are very similar in shape neglecting the small oscillations of the input force. So, in the simplified analysis we suppose that the loading is homogeneous and we proceed as in the case of quasi-static loading. The force is taken to be equal to the mean value of input and output force. 


\section{THEORETICAL SIMPLE SHEAR ANALYSIS}

The simple shear in the direction $\mathbf{e}_{1}$ in the co-ordinate system $\left(\mathbf{e}_{1}, \mathbf{e}_{2}\right)$ is defined by the relations

$$
u_{1}=\gamma(t) x_{2} \quad, \quad v_{1}=\dot{\gamma} x_{2} \text { and } u_{2}=u_{3}=v_{2}=v_{3}=0
$$

where $\gamma$ and $\dot{\gamma}$ are the plastic shear strain and shear strain rate, respectively (the strain tensor has only non-zero component $\varepsilon_{12}=\gamma$.). Using the velocity field $\mathbf{v}$ the velocity gradient $\mathbf{V}$, the rate of deformation $\mathrm{D}$ and the material spin $\omega$ can be determined in the system $\left(\mathbf{e}_{1}, \mathbf{e}_{2}\right)$ as

$$
\mathbf{V}=\frac{\dot{\gamma}}{2}\left(\begin{array}{ll}
0 & 1 \\
0 & 0
\end{array}\right) \quad \mathbf{D}=\frac{\dot{\gamma}}{2}\left(\begin{array}{ll}
0 & 1 \\
1 & 0
\end{array}\right) \quad \omega=\frac{\dot{\gamma}}{2}\left(\begin{array}{cc}
0 & 1 \\
-1 & 0
\end{array}\right)
$$

The Cauchy stress tensor $\sigma$ and the back stress $\Pi$, in the axis $x_{i}$, have the following non-zero components $\sigma_{11}, \sigma_{22}, \sigma_{12}$. and $\pi_{11}, \pi_{22}, \pi_{12}$. The presence of $\sigma_{11}$ and $\sigma_{22}$ is due to the fact that the distance between two parts of the shear device is constant during experiment i.e. $a_{o}=$ const.

Using the rate-independent constitutive relations for the adiabatic process with combined isotropickinematic hardening at moderate pressures, and neglecting the thermal expansion, the heat of elastic deformation, and the heat of internal rearrangement, we obtain the following set of equations [6] which are similar in form to those employed in problems of small strains but are applicable to the whole range of deformation processes:

$$
\breve{\mathbf{T}}=\beta \mathbf{L} \mathbf{D}-\frac{3 j \mu \beta \mathbf{D} \cdot(\overline{\mathbf{T}}-\Pi)}{\sigma_{Y}^{2} \mathcal{H}}[(\overline{\mathbf{T}}-\Pi)+\mathbf{P}]
$$

where $\beta=\rho_{o} / \rho$ is the ratio of densities in the reference and the actual configurations, $\mathbf{T}=\beta \sigma, \overline{\mathrm{T}}=\dot{\mathrm{T}}$ $\omega \mathbf{T}+\mathbf{T} \omega$ is the Zaremba-Jaumann rate, $\overline{\mathbf{T}}$ is the deviatoric part of $\mathbf{T}, \mathbf{L}$ is the fourth order tensor of elastic module, $\mu$ is the Lamé constant and $f$ is the Huber-Mises yield criterion

$$
f=\frac{3}{2}(\overline{\mathbf{T}}-\Pi) \cdot(\overline{\mathbf{T}}-\Pi)-\sigma_{Y}^{2}(\vartheta, \alpha)=0
$$

here $\sigma_{Y}$ is the yield stress in simple tension and $\alpha$ corresponds to the size of the yield surface

$$
\dot{\alpha}=(\overline{\mathbf{T}}-\Pi) \cdot \mathbf{D}^{\mathbf{P}}
$$

The shift of the yield surface is represented here by the back stress $\Pi$ for which the evolution law has the form of a linear kinematic hardening

$$
\check{\Pi}=c \mathbf{D}^{\mathbf{p}}
$$

where $c=$ const and $\mathbf{D}^{\mathbf{p}}$ is the plastic rate of deformation and $j=\left\{\begin{array}{l}1 \text { if } f=0 \text { and } \mathbf{D} \cdot(\overline{\mathbf{T}}-\Pi) \geq 0, \\ 0 \text { if } f=0 \text { and } \mathbf{D} \cdot(\overline{\mathbf{T}}-\Pi)<0 \text { or } f<0 .\end{array}\right.$

The change in the temperature field $\vartheta$ is described as

$$
\rho_{o} c_{\nu} \dot{\vartheta}=(1-\pi)(\overline{\mathbf{T}}-\Pi) \cdot \mathbf{D}^{\mathbf{p}}
$$

where $\pi$ is the scalar internal thermodynamic force conjugate with parameter $\alpha$ and $c_{v}$ is specific heat at constant volume.

The thermal expansion, the heat of elastic deformation and the heat of internal rearrangement are neglected. The first term on the right-hand side of (7) represents the rate of energy dissipation and. therefore, $\pi<1$. For numerous metals $\pi$ takes the value from 0.02 to 0.1 [6]. In the equation (3) $\exists$ is the hardening function

$$
\mathcal{H}=1+\frac{c}{2 \mu \beta}+\frac{1}{6 \mu \beta} \frac{\partial\left(\sigma_{Y}^{2}\right)}{\partial \alpha}+\frac{(1-\pi)}{6 \mu \beta \rho_{o} c_{v}} \frac{\partial\left(\sigma_{Y}^{2}\right)}{\partial \vartheta}
$$

and tensor $\mathbf{P}$ is obtained by expressing the term $\left(\omega^{\mathrm{p}} \mathbf{T}+\mathbf{T} \omega^{\mathrm{p}}\right)$ as a function of $\mathbf{D}^{\mathrm{p}}$ where $\omega^{\mathrm{p}}$ is the plastic spin.

The equation for plastic spin can be assumed to be, according to Dafalias, Paulun and Pecherski [?] and others in the following form 


$$
\omega^{\mathrm{p}}=\eta\left(\Pi \mathbf{D}^{\mathrm{p}}-\mathbf{D}^{\mathrm{p}} \Pi\right)
$$

where $\eta$ may depend on the invariants of $\mathbf{D}^{\mathrm{p}}$ and $\Pi$. Here we use the relation for the function $\eta$ occurring in this expression in the form proposed in the paper [7].

In the case of plane simple shear we have a set of four nonlinear differential equations of the first order. In the case of plasticity with kinematic hardening we also have the analytical solutions. Then $\sigma_{Y}=$ const and now from (8) we have $\mathcal{H}=1+c / 2 \mu$. Finally, after several calculations, we find the analytical solutions for the Cauchy stress components $\sigma_{11}, \sigma_{12}$ and the back stress components $\pi_{11}, \pi_{12}$ in function of shear strain. Integrating the equation (7) the temperature field can be determined.

Solutions for stresses $\sigma_{11}$ and $\sigma_{12}$ in dimensionless form vs. shear strain $\gamma$, illustrated in Fig. 4, are obtained for kinematic hardening, with $\mu=8 \cdot 10^{4} \mathrm{MPa}, c=5333,33 \mathrm{MPa}, k_{o} / \mu=0.0577, k_{o}=\sigma_{Y} / \sqrt{3}$. Results of calculations are similar to that obtained in the paper [8]. In the case of large plastic deformations the ratio $\sigma_{11} / \sigma_{12}$ and $\sigma_{22} / \sigma_{12}$ are much higher than in the case of small elastic deformations.

\section{NUMERICAL SIMULATIONS OF THE EXPERUMENT}

The program of finite element method was used to the numerical simulations of the formulated problem of quasistatic and dynamic simple shear of thin sheets. We assume the initial and boundary conditions similar to those used in the experiment. In the case of dynamic deformations a simplified supposition is introduced. The process of waves propagation in the specimen is neglected. Due to the excellent equilibrium of input and output forces and quasi-constant value in the time period $50 \mu \mathrm{s}<t<500 \mu \mathrm{s}-\mathrm{cf}$. Fig. 2, we can treated our problem as quasistatic using a rate-independent constitutive equations. The amplitude of loading is determined from the dynamic experiment. We assume that in the contact between the specimen and measure bars the force is constant in time and equal to $F_{\max }$.

In the finite element method the rectangular mesh is introduced. Deformation of the mesh in time is determined. In the same time the components of the stress tensor $\sigma_{12}$ and $\sigma_{22}=-\sigma_{11}$, the intensity of stress, the equivalent strain and the temperature field (with $\pi=0.1$ ) are determined. First, the numerical simulation was made for the quasistatic loading of the sheet made in the steel $1 \mathrm{H} 18 \mathrm{~N} 9 \mathrm{~T}$, with $\mu=8 \cdot 10^{4}$ $\mathrm{MPa} \rho=7.8 \mathrm{~g} / \mathrm{cm}^{3}$ and $\sigma_{y}=280 \mathrm{MPa}$.

Results of numerical simulation in the specimen subjected to the dynamic simple shear are shown in Fig. 5. The equivalent deformation field is shown for a half part of the shear zone of the specimen in view of the symmetry of deformation process with respect to the axis $x_{2}$. Successive sequences are presented for different values of shear strain defined as $\gamma=\Delta l(t) / a_{o}$, from $\gamma=10.2 \%$ to $\gamma=91.2 \%$. We observe for example the heterogeneity of the strain and stress fields at the free ends of the specimen at the distance less than $1.7 \%$ (accurate to $0.01 \%$ of deformation) of the total length when the strain is $30 \%$, and less than 6.6 $\%$ of the total length when the strain is $70 \%$, exactly as in the experiments.

The shear zones are manifested by a significant temperature increase. At the ends of shear zones the theoretical predicted fields of strain heterogeneities are manifested by the increase of temperature, particularly noticeable in the initial stage of shear. As the deformation continues, the line describing the position of maximum temperature departs from the shear direction. It gives evidence for the development of the macroscopic shear band, running along the specimen at a certain angle to the direction of shear.

In Fig. 3 it was also marked the position of temperature maximum in different zones of the sample obtained from numerical simulation. We observe the same tendency of the formation of the shear macrobands as in the experiment.

\section{CONCLUSIONS}

An exceptional homogeneity of the permanent strain field at finite deformations over the total length of the specimens is observed in experiments and in the results of numerical simulation. The proposed method is 
the only known test providing, in the case of a thin sheet, homogeneous stress and strain fields in both dynamic and static tests. They can be used to verify the proposed constitutive relations.

The simple shear test is particularly attractive, since the application of this type of loading path can result in large strains without the occurrence of plastic instability. But, after a certain strain of the order $70-90 \%$ the deformation becomes gradually localized. The specimen begin to be work-softened unil] fracture occurs.

Investigation of the surface temperature distribution of the sample confirm the existence of the theoretically predicted fields of strain heterogeneity. However, the quantitative modelling of the quasihomogeneous thermal effects $\vartheta(t)$ can be studied further in order to take into account the quasi-static cyclic case with moderate strains and the fatigue effects [9].
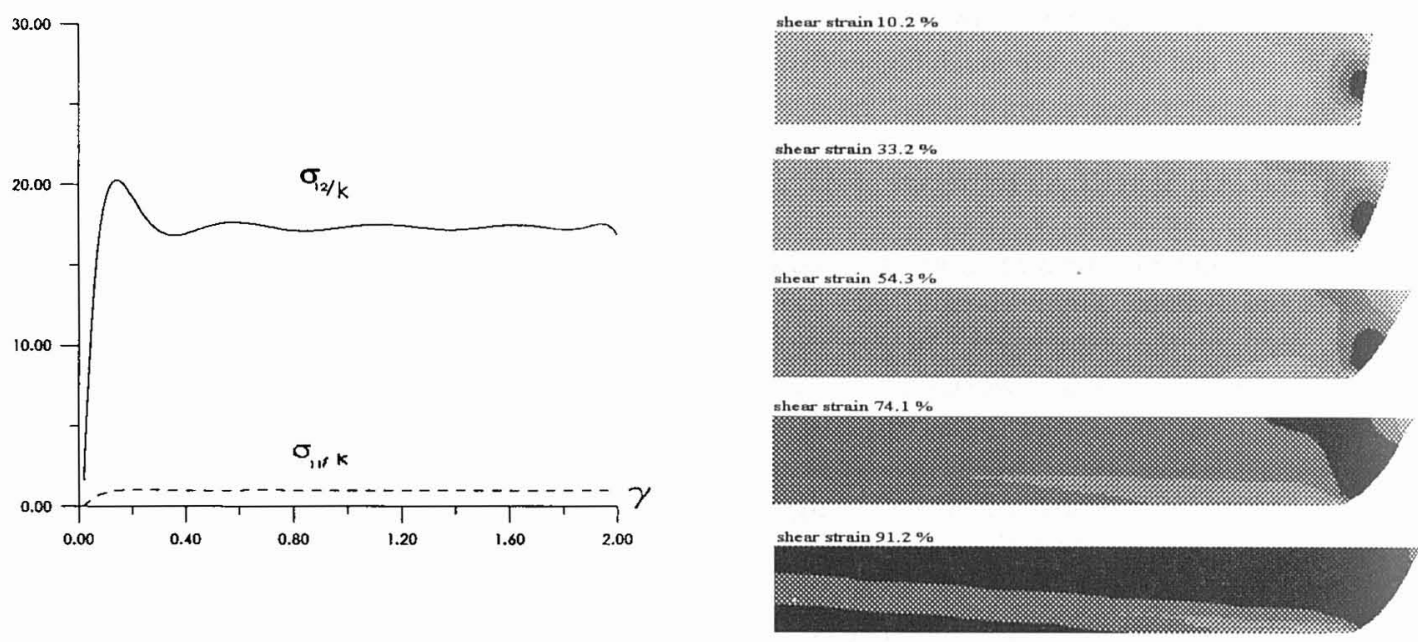

Fig. 4. Shear stress $\sigma_{l 2}$ and normal stress $\sigma_{l l}$ vs. shear strain for kinematic hardening.

Fig. 5. Numerical simulation. Equivalent deformation.

\section{Acknowledgments}

This paper is supported by the Polish Committee for Scientific Research, KBN Project No. 7T07A02608 on "Dynamic fracture of materials" and KBN Project No. 7T07A05812 on „Thermo-mechanics of large plastic deformations of steel sheets"

\section{References}

[1] Tourabi A., Wack B., Guelin P., Favier D., Pegon P., Nowacki W.K., Remarks on an experimental plane shear test and on an anisotropic elastic-plastic theory, SMIRT'93, Session L 09/4, pp.233-238.

[2] Guelin P., J. de Mécanique, 19 (1980) 217-247.

[3] Marchand A., and Duffy J., J. Mech. Phys. Solids., 36 (1988) 251-283.

[4] Gary G., and Nowacki W.K., Journal de Physique IV,Colloque C8, supplément au Journal de Physique 3 (1994) 65-70.

[5] Gadaj P.S., Nowacki W.K., Pieczyska E., Arch. Mech., 48 (1996) 779-788.

[6] Nguyen H. V., Arch. Mech., 44 (1992) 595-602.

[7] Paulun J.E., and Pecherski R.B., Arch. of Appl. Mech., 62 (1992) 376-385.

[8] Szabo L., Balla M., Int. J. Solids Structures, 3 (1989) 279-297.

[9] Tourabi A., Guelin P., Wack B., Pegon P., Favier D., Nowacki W.K., Fatigue Fract. Engng Mater. Struct. 19 (1996), 1181-1195. 